\title{
Flavonoid Content and Antioxidant Properties of Different Black Tea Infusions
}

\section{Sentkowska A and Pyrzynska $\mathrm{K}^{*}$}

University of Warsaw, Department of Chemistry, Pasteura 1, 02-093 Warsaw, Poland

${ }^{*}$ Corresponding author: Pyrzynska K, University of Warsaw, Department of Chemistry, Pasteura 1, 02-093 Warsaw, Poland, E-mail: kryspyrz@chem.uw.edu.pl

Citation: Sentkowska A, Pyrzynska K (2017) Flavonoid Content and Antioxidant Properties of Different Black Tea Infusions. J Nutr Health Sci 4(1): 104. doi: 10.15744/2393-9060.4.104

Received Date: January 25, 2017 Accepted Date: April 28, 2017 Published Date: May 22, 2017

\begin{abstract}
Tea is one of the most widely consumed beverages in the world, next only to water. Some teas are not pure varieties, but have been enhanced through additives or special processing, which allows for the design of an almost endless range of scented and flavored variants. The aromatized teas are popular due to their aroma and organoleptic properties. Tea polyphenols, especially flavonoids have attracted considerable interest because of their associated health properties. Several studies have demonstrated that tea flavonoids possess strong antioxidant activity and metal chelating properties, thus may protect cells and tissues against reactive oxygen species. In this study the content of flavonoids in the infusions of several commercially available teas (black and aromatized) was determined by HPLC-MS in the negative electrospray ionization mode. All types of tea contained epigallocatechin-3-gallate (EGCG) in the greatest amounts. Higher content of rutin was found in pure black tea infusions comparing to aromatized teas. Some teas with citrus aromas or fruits contained also other flavonoids such as naringin and hesperidin. Infusions of black teas exhibited higher antioxidant properties evaluated in FolinCiocalteu and DPPH assays.
\end{abstract}

Keywords: Black tea; Antioxidant properties; Flavonoids

\section{Introduction}

Tea is one of the most widely consumed beverages in the world, next only to water. Tea leaves primarily manufactured as green, black, white or oolong, with black tea representing approximately $80 \%$ of tea products consumed. Green tea is prepared in such a way as to preclude the oxidation of green leaf polyphenols. During black tea production oxidation is promoted, which converts monomeric phenolic (mainly catechins) into dimeric/oligomeric compounds, such as theaflavins and thearubigins, responsible for the characteristic aroma and colour of black tea. Oolong tea is a partially oxidized product. White tea is composed of only the youngest spring buds and immature leaves, thus, it represents a rare and high-priced type of tea. Some teas are not pure varieties, but have been enhanced through additives or special processing, which allows for the design of an almost endless range of scented and flavored variants. Aromatized teas are popular due to their fragrance and contain lower content of caffeine, which could inhibit calcium absorption [1]. These teas contain natural aromas as well as dry fruits or herbs, which are added to tea leaves in the last stage of processing before packing. Regular intake of tea is associated with an improved antioxidant status in vivo that may contribute to lowering the risk of coronary heart disease, atherosclerosis, reduced mutagenicity and inflammation [2,3].

Flavonoids and polyphenolic acids are the most abundant compounds in tea leaves and extracts and they are primarily responsible for the beneficial healthful properties of tea [4,5]. Antioxidative properties of phenolic compounds are manifested particularly by their abilities to inhibit free radical generation, scavenge free radicals, and chelate transition metal ions, mainly iron and copper, which are catalysts of free radical reactions [6,7]. They also prevent free radical generation by inhibiting activity of existing enzymes or by increasing the activity of enzymes with antioxidative properties, probably on the way of induction of protein molecule biosynthesis [8,9]. In this study the content of flavonoids in the infusions of several commercially available teas (black and aromatized) was determined by HPLC-MS in the negative electrospray ionization mode. Additionally, the work was completed by investigated the antioxidant properties of the prepared extracts using the Folin-Ciocalteu (FC) assay and DPPH radical scavenging activity.

\section{Materials and Methods}

\section{Chemicals}

The commercial standards of flavonoids as well as the other chemicals were purchased from Sigma (Steinheim, Germany). 
Acetonitrile was of HPLC gradient grade from Merck (Darmstadt, Germany). Ultrapure water from Milli-Q system (Millipore, Bedford, MA, USA) was used in all experiments. Stock solutions of flavonoids were prepared in methanol. Diluted mix standards were prepared with water or in methanol.

\section{Teas and infusion preparation}

All bagged teas were purchased from a local market. List of teas under study with the ingredients shown in the labels is presented in Table 1 . The tea bags were dipped into $100 \mathrm{~mL}$ of freshly boiled water for $5 \mathrm{~min}$ to represent the typical quantity consumed by tea drinkers. After the infusion time, the bags were removed and the partly turbid solutions were filtered paper after cooling to room temperature through the Whatman filter.

\begin{tabular}{|c|c|}
\hline Commercial name & Composition* (as per label) \\
\hline Citrus & $\begin{array}{l}\text { Black tea, citrus aroma }(17.5 \%) \text {, peels of } \\
\text { lemon }(1.4 \% ;) \text {, lime } \\
(1.4 \%) \text {, grapefruit }(1.4 \%) \text { and orange }(1.4 \%)\end{array}$ \\
\hline Earl Grey & $\begin{array}{l}\text { Black tea }(89.3 \%) \text {, natural aroma }(10.2 \%) \text {, } \\
\text { marigold petals }(0.3 \%) \text {, jasmine petals }(0.2 \%)\end{array}$ \\
\hline Puerh pineapple & Tea from Yunnan province, pineapple (2.8\%) \\
\hline Kashmir Valley & $\begin{array}{l}\text { Black tea }(83.2 \%) \text {, natural aroma }(8.7 \%) \text {, cin- } \\
\text { namon }(5.8 \%) \text {, rose petals }(2.3 \%)\end{array}$ \\
\hline Hyley`s & Premium black tea from Sri Lanka \\
\hline Dilmah & Premium black tea from Sri Lanka \\
\hline Saga & Premium black tea \\
\hline Venice & Premium black tea from Ceylon \\
\hline
\end{tabular}

\section{Determination of polyphenols by HPLC}

Chromatographic analysis was performed with a Shimadzu LC system consisted of a detector UV SPD 20A connected to 3200 QTRAP Mass spectrometer (Applied Biosystem/MDS SCIEX). A MS system equipped with electrospray ionization source (ESI) operated in negative-ion mode and a quadrupole mass analyser in a scan mode from 50 to $1500 \mathrm{~m} / \mathrm{z}$. Compounds were separated on KinetexTM (Phenomenex) C- 18 column $(100 \times 2.1 \mathrm{~mm}, 2,6 \mu \mathrm{m})$ with precolumn at $30^{\circ} \mathrm{C} .2 \mathrm{mM}$ formic acid (pH 2.8$)$ as eluent $\mathrm{A}$ and acetonitrile as eluent $\mathrm{B}$ were used. The mobile phase was delivered at $0.2 \mathrm{~mL} / \mathrm{min}$ in linear gradient mode: $0-5 \mathrm{~min}$. $20 \% \mathrm{~B}$, $10-15 \min 25 \%$ B, 20-25 min 30\% B, 30-31 min 90\% B, 32 min $20 \%$ B.

Compounds were identified by comparing retention time and $\mathrm{m} / \mathrm{z}$ values obtained by MS and MS 2 with the mass spectra from standards tested under the same conditions. Quantification of compounds was done from the calibration curves obtained in Multiple Reaction Mode (MRM) [10]. All the compounds showed a good linearity in the range of $0.01-40 \mathrm{mg} / \mathrm{L}$ with regression coefficients $>0.99$. Limits of detections were between 1 and $30 \mu \mathrm{g} / \mathrm{L}$ for chlorogenic acid and quercetinn, respectively.

\section{Analysis of antioxidant activity}

The Folin-Ciocalteu (FC) assay was conducted to evaluate the reducing power of the studied tea infusions. $1 \mathrm{~mL}$ of herb extract was mixed with $0.1 \mathrm{~mL}$ of FC reagent and $0.9 \mathrm{~mL}$ of water, After $5 \mathrm{~min}, 1 \mathrm{~mL}$ of sodium carbonate $(7 \%$, w/v) and $0.4 \mathrm{~mL}$ of water were added. The absorbance against a blank was measured at $765 \mathrm{~nm}$ after $30 \mathrm{~min}$. The data were expressed as gallic acid (GA) equivalent in mg/L. DPPH radical scavenging activity were examined according to the procedure by Pękal., et al. [11]. The stock solution of DPPH• $(3 \times 10-5 \mathrm{~mol} \mathrm{~L}-1)$ was prepared in methanol with acetate buffer $(0.1 \mathrm{M}, \mathrm{pH} 5.5)$ at 60:40 volume ratio. $2.4 \mathrm{~mL}$ DPPH solution was mixed with $0.1 \mathrm{~mL}$ of a given extract and immediately the change of absorbance at $539 \mathrm{~nm}$ was recorded over time against the blank. The results were expressed as Trolox (TR) equivalent (TRE).

\section{Results and Discussion}

\section{Chromatographic analysis}

Catechins (flavan-3-ols) are the dominant phenolic in tea leaves and their content varies with climate, season, horticultural practices and leaf age [12]. There is a growing evidence that these compounds have strong antioxidant activity and have numerous potentially beneficial effects on living organisms [13]. Some other phenolic compounds, such as flavonoids, also contribute to the antioxidant capacity of tea extracts. Table 2 presents the content of some flavonoids from their different subclasses determined by HPLC in the studied tea aqueous extracts.

Generally, aromatized teas contained more flavonoids in comparison to "pure" black teas. Rutin (quercetin-3-rutinoside) was found in the studied tea extracts at the highest level. Infusions of premium black teas Hyley`s and Venice contained the highest 
content of that glycoside, while Citrus tea was characterized by the lowest content of rutin. Quercetin in the form free aglycone was found at trace levels $(0.034-0.116 \mathrm{mg} / \mathrm{L})$ in all extracts. Naringin (naringenin-7-rutinoside), occurs naturally in citrus fruits, was determined only in Citrus, Kashmir Valley and Earl Grey teas. It was reported that supplementation with that glycoside significantly reduced blood glucose [14]. Luteolin from flavone family, was found only in the flavored tea infusions. Thus, these teas would support the human diet with a satisfactory source of other dietary compounds. Example of the chromatogram of Venice tea infusion obtained by LC/MS/MS is presented in Figure 1. In the infusion of this black tea, except rhamnetin, quercetin, hesperidin and rutin, also the contents of catechin and epigallocatechin-3-gallate (EGCG) were determined at $2.37 \mathrm{mg} / \mathrm{L}$ and 6.91 $\mathrm{mg} / \mathrm{L}$ level, respectively.

\begin{tabular}{|c|c|c|c|c|c|c|}
\hline Tea & Quercetin & Rhamnetin & Luteolin & Hesperidin & Naringin & Rutin \\
\hline Citrus & $0.038 \pm 0.002$ & $<$ LOD & $0.006 \pm 0.0002$ & $0.69 \pm 0.043$ & $3.51 \pm 0.099$ & $8.59 \pm 0.38$ \\
\hline Earl Grey & $0.097 \pm 0.003$ & $0.003 \pm 0.0001$ & $<$ LOD $^{* *}$ & $<$ LOD & $0.004 \pm 0.0001$ & $12.8 \pm 0.31$ \\
\hline Puerh pineapple & $0.39 \pm 0.019$ & $0.003 \pm 0.0001$ & $0.004 \pm 0.0001$ & $<$ LOD & $<$ LOD & $6.30 \pm 0.29$ \\
\hline Kashmir Valley & $0.116 \pm 0.004$ & $0.011 \pm 0.0005$ & $0.015 \pm 0.001$ & $<$ LOD & $0.009 \pm 0.0003$ & $10.8 \pm 0.40$ \\
\hline Hyley`s & $0.065 \pm 0.009$ & $<$ LOD & $<$ LOD & $<$ LOD & $<$ LOD & $25.7 \pm 0.072$ \\
\hline Dilmah & $0.035 \pm 0.009$ & $<$ LOD & $<$ LOD & $<$ LOD & $<$ LOD & $13.5 \pm 0.34$ \\
\hline Saga & $<$ LOD & $0.009 \pm 0.001$ & $<$ LOD & $<$ LOD & $<$ LOD & $13.4 \pm 0.41$ \\
\hline Venice & $0.034 \pm 0.008$ & $0.012 \pm 0.0005$ & $<$ LOD & $0.025 \pm 0.005$ & $<$ LOD & $25.1 \pm 0.69$ \\
\hline
\end{tabular}

${ }^{*}$ Mean $\pm \mathrm{SD}(\mathrm{n}=3)$, expressed in $\mathrm{mg} / \mathrm{L} ;{ }^{* *} \mathrm{LOD}-$ limit of detection

Table 2: Content ${ }^{\star}$ of selected flavonoids in studied tea infusions

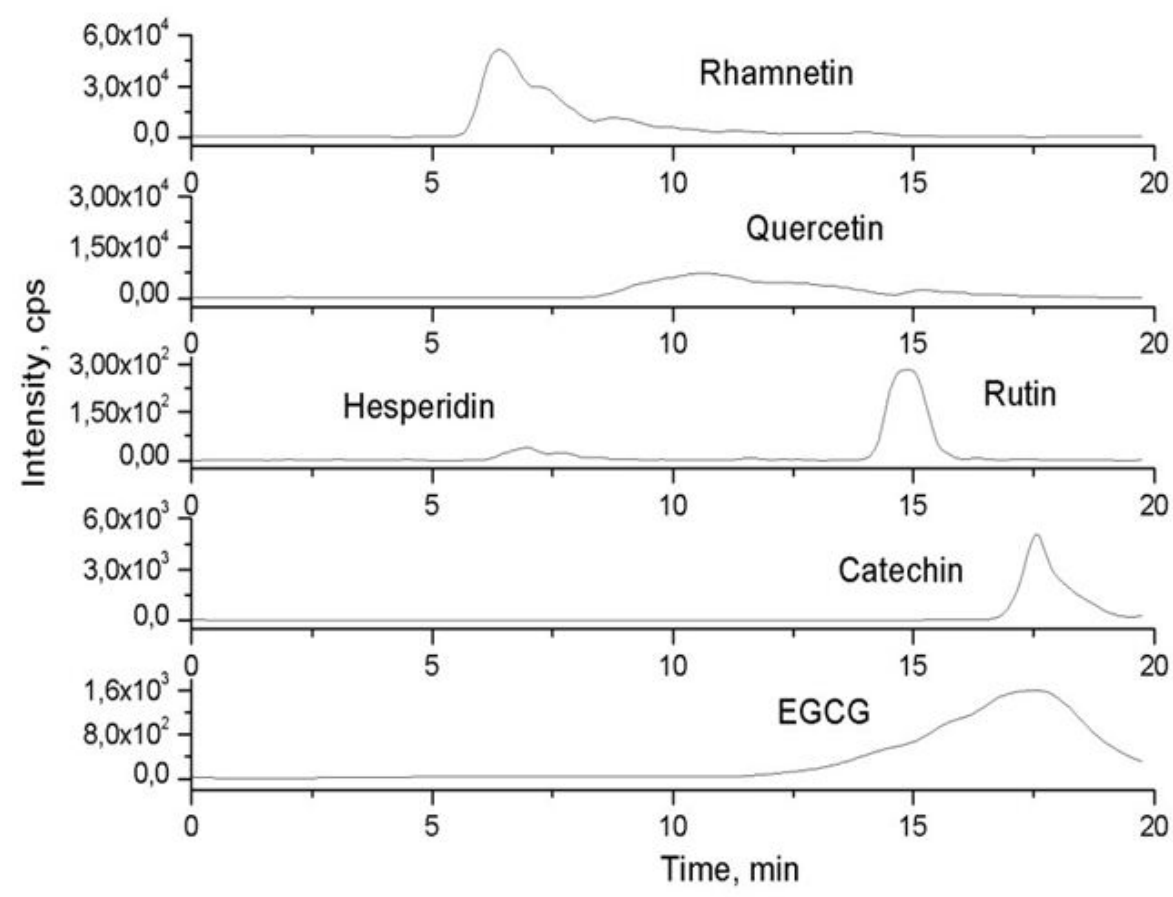

Figure 1: Extracted ion chromatograms of flavonoids in the extract of Venice tea

\section{Antioxidant activity of tea infusions}

The reducing power of the studied tea infusions was evaluated by Folin-Ciocalteu assay. This assay, very often called in the published papers as the "total phenolic content" is based on a single electron transfer reaction and it is assumed that the antioxidant activity is equal to the reducing capacity of the sample. However, FC reagent is not specific only for phenolic compounds as well some inorganic substances to give elevated apparent phenolic content $[15,16]$. The obtained results, expressed as gallic acid (GA) equivalent in $\mathrm{mg} / \mathrm{L}$, are presented in Table 3. The reducing power of the extracts of studied teas were in the range of $345-1119$ mg GA/L. Significantly higher values were determined for "pure" black tea infusions (830-1119 mg GA/L) in comparison to aromatized teas (345-701 mg GA/L). Black teas contain higher content of rutin (Table 2) as well as catechin and their derivatives [10]. They may contain also, except determined flavonoids, other compounds with reducing properties.

Antioxidant capacity of the studied extracts was also assessed on the basis of their scavenging effect on the 1,1-diphenyl-2pirylhydrazyl (DPPH) radicals. The decay slope and the absorbance level reached by the remaining DPPH radicals vary with different types of studied plants (Figure 2). During the first few minutes of the reaction, significant decrease in the absorbance of 
DPPH was observed, followed by slow subsequent disappearance of the reagent. This fast step essentially refers to the electrontransfer process from $\mathrm{B}$ ring $\left(\mathrm{C}^{\prime}{ }^{-}-\mathrm{OH}\right.$ and $\left.\mathrm{C} 4{ }^{\circ}-\mathrm{OH}\right)$ of flavonoid molecules to $\mathrm{DPPH}$ radical and latter kinetic reflects the remaining activity of the oxidation-degradation products.
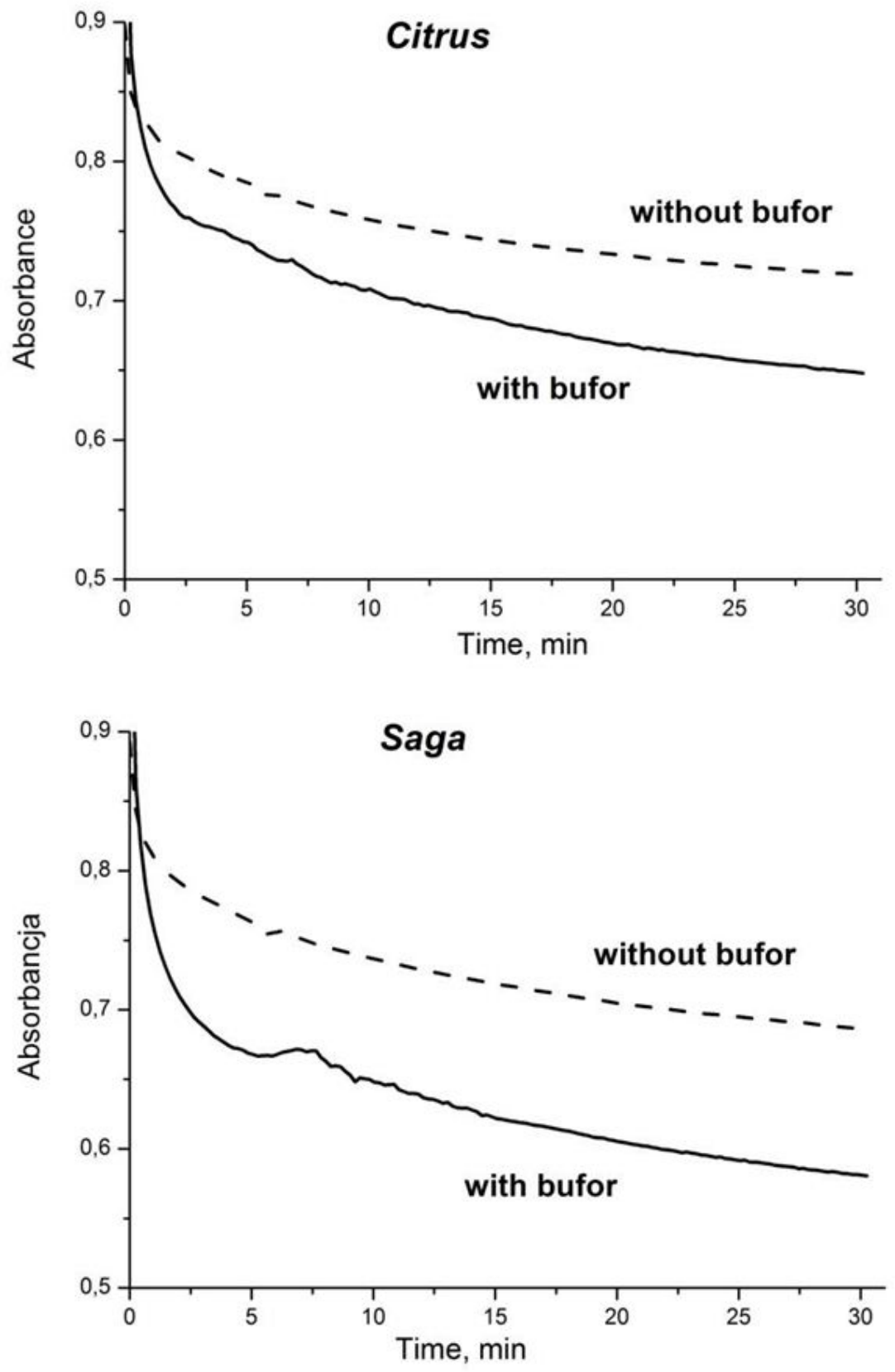

Figure 2: The kinetic curves of scavenged DPPH by the extracts of Citrus and Saga teas

DPPH assay is one of the most popular and frequently employed methods to test the ability of compounds to act as free radical scavengers and several protocols have been followed with different reaction time, solvents, $\mathrm{pH}$ and compounds used as an antioxidant standard $[17,18]$. As plant extracts can differ quantitatively and qualitatively not only in the composition of antioxidants, but also of natural acids, thus in consequence, extracts $\mathrm{pH}$ can be different. In the original Blois paper [19] regarding the use of the DPPH method, it was suggested that the system should be maintained at pH in the range 5.0-6.5 by using acetate buffers. However, these conditions were abandoned in the latter current practice. Figure 2 presents the kinetic curves of scavenged DPPH by the extracts of some studied tea infusions when buffered and non-buffered reagent was used. The decrease of hydrogen ion concentration leads to the increase of the reaction rate between DPPH and the components present in the extracts. which confirms earlier report [20].

The results for the scavenging effect on DPPH radicals measured after $30 \mathrm{~min}$ of reaction, express as trolox equivalent, (mmol TR/L) are presented in Table 3. The highest value was determined for Saga extract, although infusion of this tea exhibited the lowest reducing power evaluated by FC assay amongst all studied "pure" black teas. 


\begin{tabular}{|c|c|c|}
\hline Tea & $\begin{array}{c}\text { FC } \\
(\mathbf{m g ~ G A} / \mathbf{L})\end{array}$ & $\begin{array}{c}\text { DPPH } \\
(\mathbf{m m o l} \text { TR/L) }\end{array}$ \\
\hline Citrus & $345 \pm 12.9$ & $0.35 \pm 0.01$ \\
\hline Earl Grey & $701 \pm 28.1$ & $0.43 \pm 0.02$ \\
\hline Puerh pineapple & $507 \pm 14.0$ & $0.39 \pm 0.02$ \\
\hline Kashmir Valley & $546 \pm 19.7$ & $0.44 \pm 0.03$ \\
\hline Hyley`s & $831 \pm 37.1$ & $0.55 \pm 0.03$ \\
\hline Dilmah & $1119 \pm 40.7$ & $0.56 \pm 0.02$ \\
\hline Saga & $830 \pm 33.4$ & $0.87 \pm 0.04$ \\
\hline Venice & $1147 \pm 38.7$ & $0.56 \pm 0.03$ \\
\hline
\end{tabular}

Mean \pm SD $(n=3)$

Table 3: Antioxidant activity of tea extracts evaluated by FC and DPPH assays.

\section{Conclusion}

The results of this research have provided an insight into the differences in antioxidant properties of different black teas with or without any additives. Spectrophotometric methods used in this work provided data confirming protective role of tea in the fight against antioxidative stress. Different kind of tea additives can also influence the antioxidative properties of tea infusion. Their natural origin enriches such infusion in additional compounds.

\section{Acknowledgment}

The authors would like to thank the Structural Research Laboratory (SRL) at the Department of Chemistry of University of Warsaw for making possible HPLC-MS measurements. SRL has been established with financial support from European Regional Development Found in the Sectorial Operational Programme "Improvement of the competitiveness of Enterprises, years 2004 -2005” project no: WPK_1/1.4.3./1/2004/72/72/165/2005/U.

\section{References}

1. Aoshima A, Hirata S, Ayabe S (2007) Antioxidative and anti-hydrogen peroxide activities of various herbal teas. Food Chem 391: 617-22.

2. Sharangi AB (2009) Medicinal and therapeutic potentialities of tea (Camelia sinensis L.) A review. Food Res Inter 42: 529-35.

3. Pan MH, Lai CS, Ho TC (2010) Anti-inflammatory activity of natural dietary flavonoids. Food Funct. 1: 15-31.

4. Khan N, Mykhtar H (2007) Tea polyphenols for health promotion. Life Sci. 81: 519-33.

5. Acosta-Estrada BA, Gutierrez-Uribe JA, Serna-Saldivar SO (2014) Bound phenolics in foods, a review. Food Chem. 152: 46-55.

6. Wang H, Provan GI, Helliwell K (2000) Tea flavonoids: their functions, utilization and analysis. Trends Food Sci Technol. 11: 152-60.

7. Soto-Vaca A, Gutierrez AA, Losso JN, Xu Z, Finley JW (2012) Evolution of phenolic compounds from color and flavor problems to health benefits. J. Agric. Food Chem. 60: 6658-77.

8. Spencer JPE, Vauzour D, Rendeiro C (2009) Flavonoids an cognition. The molecular mechanisms underlying their behavioural effects. Arch. Biochem. Biophys. 492: $1-9$.

9. Santhakumar AB, Bulmer AC, Singh J (2014) A review of the mechanisms and effectiveness of dietary polyphenols in reducing oxidative stress and thrombotic risk. J Hum Nutr Diet 27: 1- 21.

10. Pękal A, Drożdż P, Biesaga M, Pyrzynska K (2011) Evaluation of the antioxidant properties of fruit and flavored black tea. Eur. J. Nutr. 50: 681-8.

11. Pękal A, Pyrzynska K (2015) Effect of pH and metal ions on DPPH radical scavenging activity of tea. Inter J Food Sci Nutr. 66: 58-62.

12. Li S, Lo CY, Pan MH, Lai CS, Ho CT (2013) Black tea: chemical analysis and stability. Food Funct. 4: 10-18.

13. Lambert JD, Elias RJ (2010) The antioxidant and pro-oxidant activities of green tea polyphenols: A role in cancer prevention. Arch. Biochem. Biophys. 501: 65-72.

14. Jung UJ, Lee MK, Jeong KS, Choi MS (2004) The hypoglycemic effects of hesperidin and naringin are partly mediated by hepatic glucose-regulating enzymes in C57BL/KsJ-db/db mice. J Nutrit 134: 2499-503.

15. Everette JD, Bryant QM, Green AM, Abbey YA, Wangila GW, et al. (2010) Through study of reactivity of various compound classes toward the Folin-Ciocalteu reagent. J Agric Food Chem. 58: 8139-44.

16. Castro-Alves VC, Cordenunsi BR (2015) Total soluble phenolic compounds quantification is not as simple as it seems. Food Anal Meth. 8: 873-84.

17. Mishra K, Ojha H, Chaudhury NK (2012) Estimation of antiradical properties of antioxidants using DPPH assay: A critical review and results. Food Chem. 130: $1036-43$.

18. Pyrzynska K, Pękal A (2013) Application of free radical diphenylpicrylhydrazyl (DPPH) to estimate the antioxidant capacity of food samples. Anal Meth. 5: 4288-95.

19. Blois MS (1958) Antioxidant determination by the use of a stable free radical. Nature 181: 1199-200.

20. Dawidowicz AL, Olszowy M (2012) Mechanism change in estimating of antioxidant activity of phenolic compounds. Talanta 97: $312-7$. 


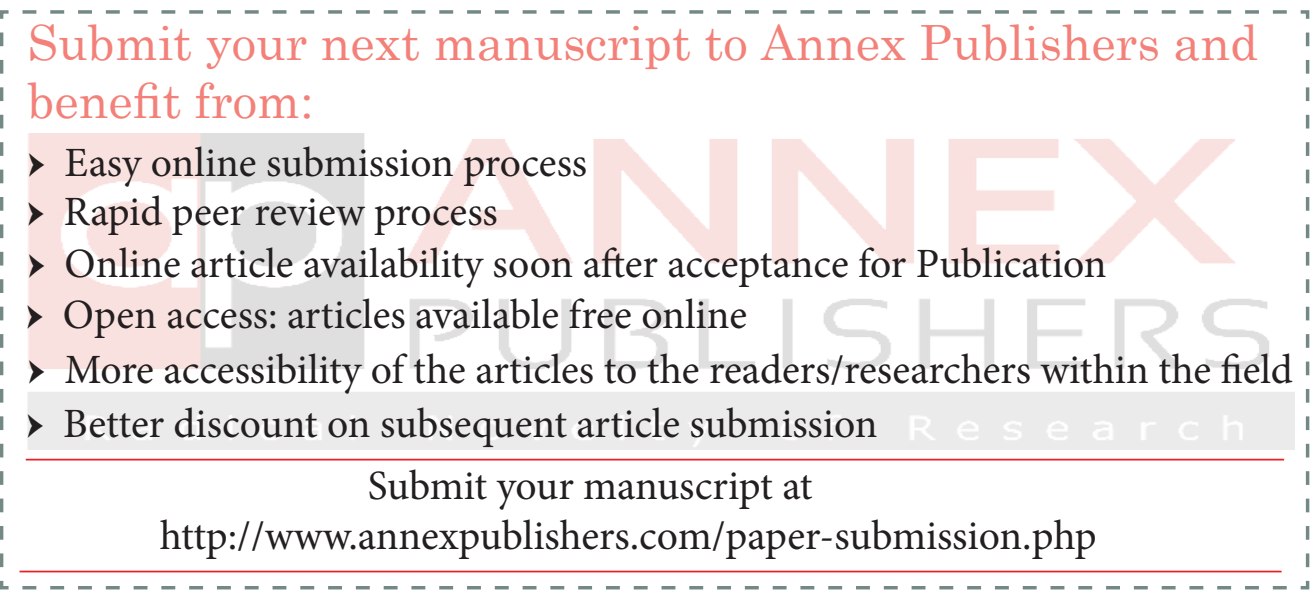

